\title{
Ausgewählte Methoden der Prozessverbesserung
}

\author{
Klaus D. Bösing
}

\section{Einleitung}

Heute werden nur diejenigen Unternehmen im harten Konkurrenzkampf überleben, denen es gelingt, ihre Geschäftsprozesse in geeigneter Weise zu organisieren und zu optimieren. Mit Hilfe des Geschäftsprozessmanagements können dabei strategisch relevante Geschäftsprozesse im Unternehmen nachhaltig verbessert werden.

Grundsätzlich gibt es zwei Vorgehensweisen, um die Leistung von Geschäftsprozessen zu optimieren:

- Prozesserneuerung

- Prozessverbesserung

Während die Prozesserneuerung nur in besonderen Situationen zur Anwendung kommt, wird die Prozessverbesserung kontinuierlich durchgeführt.

$\mathrm{Zu}$ den bekanntesten Methoden der Prozesserneuerung gehört das Business Reengineering von M. Hammer und J. Champy [3], die in der Literatur auch als Business Process Reengineering (BPR) bezeichnet wird. Ziel dieser Methode ist es, durch radikale Neugestaltung von entscheidenden Geschäftsprozessen des Unternehmens die Verbesserung von Kosten, Zeit und Qualität, um die Wettbewerbsfähigkeit am Markt zu sichern (vgl. u. a. [1]).

Bezüglich der Prozessverbesserung zählen

- Total Cycle Time (TCT),

- Kaizen und

- Six Sigma

zu den seit vielen Jahren mit Erfolg angewandten Methoden. Ziel dieser Methoden ist es, die Prozesse kontinuierlich zu verbessern und somit die Prozessleistung zu erhöhen.

\section{Prozessverbesserungsmethoden}

Um die Ursachen von ineffizienten und ineffektiven Geschäftsprozessen zu reduzieren, gehen alle oben genannten Methoden nach dem Problemlösungskreislauf vor (Abb. 1):

1. Probleme identifizieren

2. Probleme analysieren

3. Ursachen beseitigen

4. Maßnahmen prüfen

5. Lösung standardisieren

Während die TCT-Methode den Gesamtprozess und die Ebenen der Teilprozesse betrachtet, konzentriert sich Kaizen auf die Ebene einzelner Prozess- und Arbeitsschritte. Bei Six Sigma können sich die Verbesserungen auf alle Ebenen beziehen. Alle drei Methoden haben gemeinsam, dass sie kontinuierlich durchgeführt werden und auf dem Prinzip der kleinen Schritte beruhen.

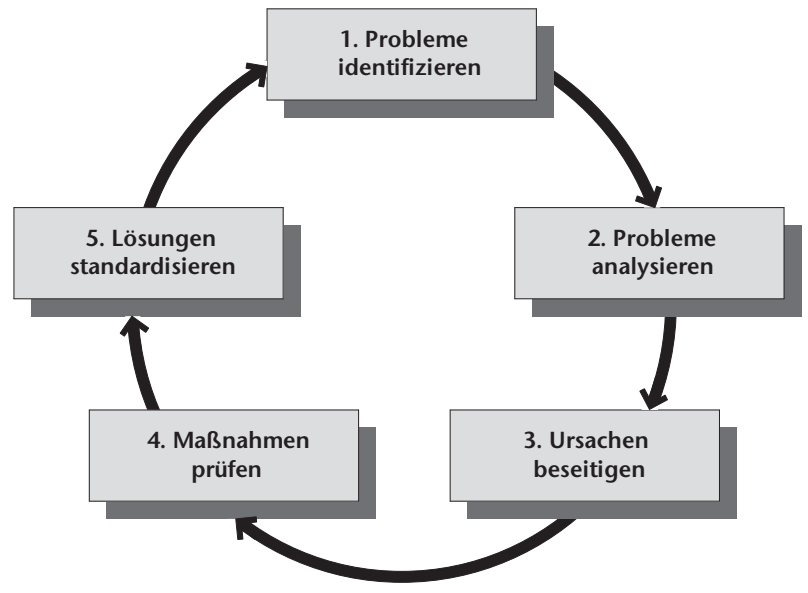

Abb. 1: Prozessverbesserung in 5 Schritten (vgl. [5])

\subsection{TCT-Methode}

Die TCT-Methode stammt aus den USA. Im Vordergrund dieser Methode steht die Reduzierung der Prozesszeit. Ziel ist es, die Prozesszeit so zu reduzieren, dass gleichzeitig die anderen Leistungsparameter wie Prozesskosten, Prozessqualität und Termintreu optimiert werden (vgl. [2]).

Die wesentlichen Merkmale der TCT-Methode sind (vgl. u. a. [5]):

- Sondieren von Barrieren, die den Ablauf von Geschäftsprozessen behindern,

- Eliminieren von Barrieren und Ersatzprozessen,

- Messen der Wirkungen bzgl. der Beseitigung von Barrieren über die Leistungsparameter Prozesszeit, Prozessqualität und Termintreue,

- Vergleich der gemessenen Ist-Werte mit den Ziel-Werten für Prozesszeit, Prozessqualität und Termintreue.

Probleme, die einen Prozess behindern, werden als Barrieren bezeichnet. Die TCT-Methode differenziert zwischen

- Kulturbarrieren (z. B. nicht klar formulierte Ziele),

- Prozessbarrieren (z. B. Wartezeiten) und

- Sachbarrieren (z. B. fehlende Informationen).

Ersatzprozesse werden durch unerwartete Ereignisse ausgelöst und sind mit Kosten verbunden. Sie dienen dazu, Irrtümer und Fehler zu beseitigen. Ein Beispiel für einen Ersatzprozess ist die Fehlerbehebung bei der Programmierung. Gäbe es keine Fehler, dann würde auch kein Ersatzprozess entstehen und somit auch keine zusätzlichen Kosten.

Die Wirkungen, die mit der TCT-Methode verbunden sind, sind beispielsweise (vgl. u. a. [5]):

- die Leistungssteigerung der Prozesse auf der Ebene der Geschäftsprozesse,

- die Steigerung der Identifikation und Motivation der Mitarbeiter sowie

- die Intensivierung der internen und externen Kundenorientierung 


\subsection{Kaizen-Methode}

Der japanische Begriff Kaizen setzt sich aus den Worten Kai für „Veränderung“ und „Wandel“ sowie Zen für „zum Besseren" zusammen und bedeutet die ständige und systematische Verbesserung von Prozessen in kleinen Schritten.

Die Kaizen-Methode kann als Bottom-Up Vorgehensweise charakterisiert werden, deren Ziel es ist, auf der Ebene einzelner Prozesse und Arbeitsschritte die Leistung von Geschäftsprozessen kontinuierlich zu steigern.

Die Philosophie der Kaizen-Methode betont vor allen den Menschen und sein Potenzial zur Problemlösung. Daher richten sich die Investitionen eines Unternehmens vielmehr auf das Humankapital und weniger auf Technologien. Personalkosten werden als Investition in die Zukunft und nicht als Kostenfaktor gesehen.

Das Ziel der Kaizen-Methode ist die Beseitigung von Verschwendung. „Verschwendung ist alles, was für die Kunden keinen Nutzen hat und wofür sie nicht bereit sind, zu zahlen" [5]. Es wird also alles, was nicht der direkten Wertschöpfung dient, als Verschwendung betrachtet. Verschwendungen können beispielsweise sein:

- unnötige Wartezeiten

- entbehrliche Arbeitsprozesse

- unklare Zielsetzungen

- mangelnde Koordination sowie

- mangelhafte oder überflüssige Informationen

Für die Beseitigung von Verschwendungen stellt die Kaizen-Methode folgende Werkzeuge und Methoden zur Verfügung:

- 5-S-Vorgehen (5-A-Vorgehen)

- 7-W- und 3-MU-Checklisten

- PDCA-Verbesserungszyklus (Deming-Rad)

- sieben alte Werkzeuge und

- sieben neue Werkzeuge

Das 5-S-Vorgehen (5-A-Vorgehen) beschreibt eine einfache Vorgehensweise, mit der schnell Verbesserungen erzielt werden. Dabei stehen

- Seiri für Ordnung schaffen (Aussortieren),

- Seiton für alles am richtigen Platz (Aufräumen),

- Seiso für Sauberkeit (Arbeitsplatz sauber halten),

- Seiketsu für persönlicher Ordnungssinn (Anordnen zur Regel machen) und

- Shitsuke für Disziplin (Alle Punkte einhalten und ständig verbessern).

Die Checklisten 7-W:

- Wer macht es?

- Warum macht er es?

- Wann wird es gemacht?

- Wo soll es getan werden?

- Wie wird es gemacht?

- Wieso wird es nicht anders gemacht?

sowie 3-MU:

- Muda (Verschwendung)

- Muh (Überlastung)

- Mura (Abweichung)

dienen der regelmäßigen Überprüfung, wobei sich die letzten drei Punkte auf die Mitarbeiter, Technik und Methode beziehen.

Ein weiteres Werkzeug ist der PDCA-Verbesserungszyklus, der auch als Plan-Do-Check-Act-Zyklus (Abb. 2) oder nach dem Erfinder als Deming-Kreis bezeichnet wird. Hierbei wird ein Kreislauf zur Verbesserung beschrieben. Er beginnt mit der Problemerkennung der gegenwärtigen Situation, um einen Plan zur Verbesserung zu formulieren. Dieser Plan wird nach der Fertigstellung umgesetzt und es wird überprüft, ob die erwarteten Ergebnisse erzielt wurden. Bei einer positiven Bewertung werden diese Maßnahmen als Standard definiert. Dieser Standard unterliegt dann wiederum einem erneuten Verbesserungszyklus.

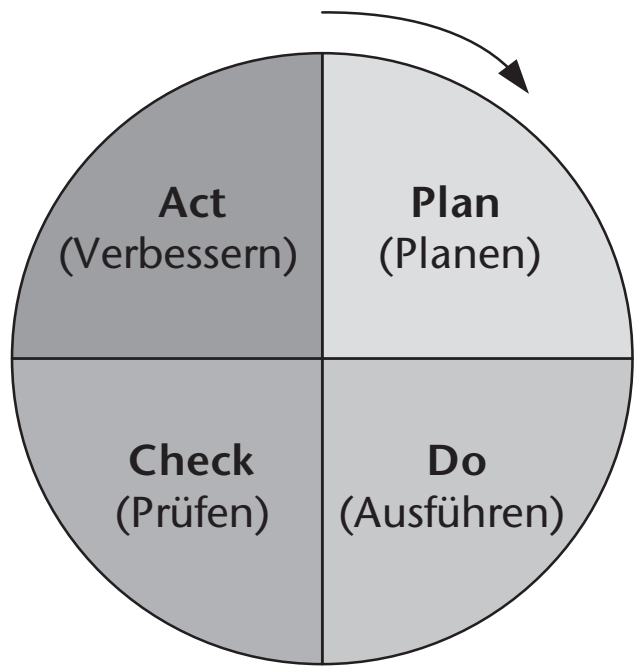

Abb. 2: Plan-Do-Check-Act-Zyklus

Können Probleme auf der Grundlage von statistischen Daten ermittelt werden, so werden die „sieben alten Werkzeuge" eingesetzt. Hierzu gehören:

- Ishikawa-Diagramm (Ursache-Wirkungs-Diagramm)

- Pareto-Diagramm (ABC-Analyse)

- Histogramm

- Streuungsdiagramm

- Kontrollkarte

- Kurvendiagramm

- Prüfformular

Wenn keine statistischen Daten zur Verfügung stehen, werden entsprechende Daten mit Hilfe der „sieben neuen Werkzeuge“ ermittelt. Dazu zählen:

- Beziehungsdiagramm

- Affinitätsdiagramm

- Baumdiagramm

- Matrixdiagramm

- Matrixdiagramm zur Datenanalyse

- Diagramm zur Entscheidungsfindung

- Pfeildiagramm

$\mathrm{Zu}$ erwähnen sind noch die Begriffe Cross-Functional Management und Policy Deployment.

Unter Cross-Functional Management (Funktionsüberschneidendes Management) wird die abteilungs- und bereichsübergreifende Koordination von Aktivitäten eines Unternehmens verstanden. Eine besondere Bedeutung bekommt hier die interdisziplinäre Zusammenarbeit einzelner Fachabteilungen.

Unter Policy Deployment wird die Durchgängigkeit der Unternehmenspolitik verstanden. Es ist ein Planungssystem, mit dem die ständigen Verbesserungen in einem Unternehmen umgesetzt werden. Während mit dem CrossFunctional Management die horizontale Integration von 
Aktivitäten eines Unternehmens unterstützt wird, liegt der Schwerpunkt hier auf der vertikalen Integration von Aktivitäten.

Die Kaizen-Methode ist stark auf die Fähigkeit und Bereitschaft der Mitarbeiter eines Unternehmens ausgerichtet. Die Wirkungen sind mit denen der TCT-Methode vergleichbar.

KVP (Kontinuierlicher Verbesserungsprozess) ist die europäische Variante der Kaizen-Methode. Beispielsweise wurde KVP von VW zu KVP im Quadrat weiterentwickelt (vgl. [4]).

\subsection{Six-Sigma-Methode}

Six Sigma ist ein Begriff aus der Statistik und steht als Synonym für Null-Fehler-Qualität. Das bedeutet, dass in einem Prozess, der Six Sigma erfüllt, bei einer Million Möglichkeiten nur 3,4 fehlerhafte Ergebnisse entstehen. Dies entspricht einem Qualitätsgrad von 99,9997 \%.

Ursprünglich wurde Six Sigma für Produktionsprozesse entwickelt, während es heute auch in Dienstleistungsprozessen erfolgreich eingesetzt wird. Zentrale Ziele von Six Sigma sind Kosteneinsparungen und die Verbesserung der Qualität.

Six Sigma ist eine empirische, datenorientierte Methode, bei der die Verringerung der Varianz, also die Abweichung der Prozessergebnisse vom Ziel-Wert, im Mittelpunkt steht. Im Mittelpunkt von Six Sigma steht der Prozessverbesserungszyklus (DMAIC-Cycle), der aus 5 Phasen besteht (Abb. 3).

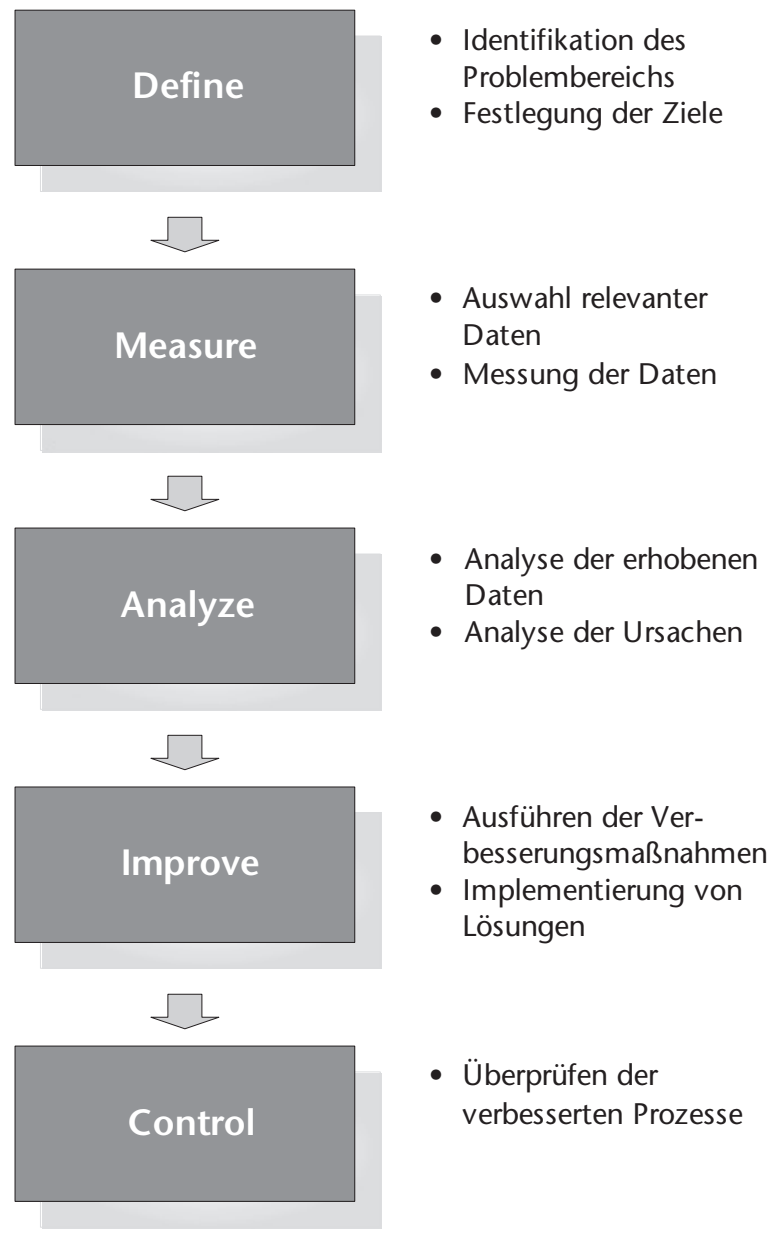

Abb. 3: DMAIC-Zyklus
Maßeinheiten bei Six Sigma sind Fehler pro Million Möglichkeiten (FpMM) und die Varianz Sigma $(\sigma)$. Fehler sind Abweichungen vom Ziel-Wert. Mit Hilfe einer Umrechnungstabelle kann anhand der Fehleranzahl der Wert für $\sigma$ ermittelt werden. Ziel ist es, eine Varianz von $6 \sigma \mathrm{zu}$ erreichen.

Ein Vorteil von Six Sigma ist, dass man aufgrund der Normalisierung der Fehlerrate einen Wert erhält, mit dem sich unterschiedliche Geschäftsprozesse einer Organisation vergleichen lassen. Für die Normalisierung von Werten wird die gemessene Fehlerrate auf eine Million Fehlermöglichkeiten normalisiert.

Bei der Vorgehensweise der Prozessverbesserung wird bei Six Sigma zunächst ein einzelner Prozessschritt betrachtet. Der $\sigma$-Wert für den Gesamtprozess errechnet sich dann aus dem Produkt der $\sigma$-Werte der einzelnen Prozessschritte.

Die wesentlichen Werkzeuge der Six-Sigma-Methode zur Verbesserung von Prozessen sind mit den „sieben alten Werkzeugen" der Kaizen-Methode identisch, also

- Ishikawa-Diagramm (Ursache-Wirkungs-Diagramm)

- Pareto-Diagramm (ABC-Analyse)

- Histogramm

- Streuungsdiagramm

- Kontrollkarten

- Kurvendiagramm

- Prüfformular

Ohne Six Sigma liegen nach Schätzungen die meisten Prozesse in der Industrie gegenwärtig bei ca. 3 bis $4 \sigma$. Mit herkömmlichen Prozessverbesserungsmethoden wird es nur schwer für möglich gehalten, eine signifikante Verbesserung in Richtung $5 \sigma$ oder mehr zu erreichen. Zur Veranschaulichung ist in Abbildung 4 das Verhältnis von $\sigma$, den Fehlern pro 1 Million Möglichkeiten (FpMM) und dem Qualitätsgrad gegenübergestellt.

\begin{tabular}{|c|c|c|}
\hline$\sigma$ & FpMM & Qualitätsgrad \\
\hline 1 & 691.462 & $30,8538 \%$ \\
\hline 2 & 308.538 & $69,1483 \%$ \\
\hline 3 & 66.807 & $93,3193 \%$ \\
\hline 4 & 6.210 & $99,3790 \%$ \\
\hline 5 & 233 & $99,9767 \%$ \\
\hline 6 & 3,4 & $99,9997 \%$ \\
\hline
\end{tabular}

Abb. 4: Verhältnis von $\sigma, F p M M$ und Qualitätsgrad

\section{Zusammenfassung}

In Abbildung 5 sind noch mal die Unterschiede und Gemeinsamkeiten der drei Methoden TCT, Kaizen und Six Sigma zusammengefasst.

\begin{tabular}{|c|c|c|}
\hline TCT & Kaizen & Six Sigma \\
\hline $\begin{array}{l}\text { - Teilprozesse } \\
\text { - Geschäftsprozesse }\end{array}$ & $\begin{array}{l}\text { - Arbeitsschritte } \\
\text { - Prozessschritte }\end{array}$ & $\begin{array}{l}\text { - Prozessschritte } \\
\text { - Gesamtprozesse }\end{array}$ \\
\hline $\begin{array}{l}\text { - Beseitigung von } \\
\text { Barrieren } \\
\text { - Reduzierung der } \\
\text { Prozesszeit }\end{array}$ & $\begin{array}{l}\text { - Beseitigung von } \\
\text { Verschwen- } \\
\text { dungen }\end{array}$ & \begin{tabular}{|c|}
- Reduzierung \\
der Varianz \\
- Erreichung von \\
\\
$\sigma=3,4$ FpMM
\end{tabular} \\
\hline $\begin{array}{l}\text { - u. a. Ursache- } \\
\text { Wirkungs- } \\
\text { Diagramm }\end{array}$ & $\begin{array}{l}\text { - sieben alte und } \\
\text { neue Werkzeuge } \\
\text { - Checklisten }\end{array}$ & $\begin{array}{l}\text { - sieben alte } \\
\text { Werkzeuge }\end{array}$ \\
\hline
\end{tabular}

Abb. 5: Vergleich der Methoden 
Die wesentlichen Unterschiede der drei Methoden liegen in der Zielsetzung. Bei der TCT-Methode liegen die Ziele in der Beseitigung von Barrieren, den Ersatzprozessen und der Reduzierung der Zykluszeit. Bei der Kaizen-Methode sind die Ziele ähnlich. Hier werden ebenfalls alle Aktivitäten eliminiert, die keinen Wertzuwachs hervorrufen. Six Sigma verfolgt die Strategie der Reduzierung von Zielabweichungen.

\section{Literatur}

[1] Becker, J.; Kugeler, M.; Rosemann, M. (Hrsg.): Prozessmanagement, 4. Auflage, Springer-Verlag, Berlin, Heidelberg, New York 2003

[2] Bösing, K. D.: Business Process Performance Management, Forschungsbericht WS 2004/2005, Technische Fachhochschule Wildau, Wildau 2005

[3] Hammer, M.; Champy, J.: Business Reengineering, CampusVerlag, Frankfurt, New York 1994

[4] Jetter, W.: Performance Management, Schäffer-Poeschel Verlag, Stuttgart 2000

[5] Schmelzer, H. J.; Sesselmann, W.: Geschäftsprozessmanagement in der Praxis, 3. Auflage, Carl Hanser Verlag, München, Wien 2003

\section{Autor}

Prof. Dr. Klaus D. Bösing

Technische Fachhochschule Wildau

Fachbereich Betriebswirtschaft/Wirtschaftsinformatik

Fachgebiet Software Engineering

Tel. +49 3375 508-952

boesing@tfh-wildau.de 